\title{
BUGJEFF311.BOLIB (JEFF-3.1.1) and BUGENDF70.BOLIB (ENDF/B-VII.0) - Generation Methodology and Preliminary Testing of two ENEA-Bologna Group Cross Section Libraries for LWR Shielding and Pressure Vessel Dosimetry
}

\author{
Massimo Pescarini ${ }^{1, \text { a }}$, Valentin Sinitsa ${ }^{2}$, Roberto Orsi ${ }^{1}$, and Manuela Frisoni ${ }^{1}$ \\ ${ }^{1}$ ENEA, CR "E. Clementel”, via Martiri di Monte Sole 4, 40129, Italy \\ ${ }^{2}$ NRC “Kurchatov Institute”, 1, Academica Kurchatova pl., 123182 Moscow, Russian Federation
}

\begin{abstract}
Two broad-group coupled neutron/photon working cross section libraries in FIDO-ANISN format, dedicated to LWR shielding and pressure vessel dosimetry applications, were generated following the methodology recommended by the US ANSI/ANS-6.1.2-1999 (R2009) standard. These libraries, named BUGJEFF311.BOLIB and BUGENDF70.BOLIB, are respectively based on JEFF-3.1.1 and ENDF/B-VII.0 nuclear data and adopt the same broad-group energy structure $(47 \mathrm{n}+20 \gamma)$ of the ORNL BUGLE-96 similar library. They were respectively obtained from the ENEABologna VITJEFF311.BOLIB and VITENDF70.BOLIB libraries in AMPX format for nuclear fission applications through problem-dependent cross section collapsing with the ENEA-Bologna 2007 revision of the ORNL SCAMPI nuclear data processing system. Both previous libraries are based on the Bondarenko self-shielding factor method and have the same AMPX format and fine-group energy structure $(199 \mathrm{n}+42 \gamma)$ as the ORNL VITAMIN-B6 similar library from which BUGLE-96 was obtained at ORNL. A synthesis of a preliminary validation of the cited BUGLE-type libraries, performed through 3D fixed source transport calculations with the ORNL TORT-3.2 $\mathrm{S}_{\mathrm{N}}$ code, is included. The calculations were dedicated to the PCA-Replica 12/13 and VENUS-3 engineering neutron shielding benchmark experiments, specifically conceived to test the accuracy of nuclear data and transport codes in LWR shielding and radiation damage analyses.
\end{abstract}

\section{Introduction}

The ENEA-Bologna Nuclear Data Group generated the NEA-1866/BUGJEFF311.BOLIB [1] and the NEA-1870/BUGENDF70.BOLIB [2] multi-group coupled neutron/photon working cross section libraries in FIDO-ANISN format, respectively based on the OECD-NEADB JEFF-3.1.1 [3, 4] and the US ENDF/B-VII.0 [5] evaluated nuclear data libraries. These twin libraries dedicated to LWR radiation shielding and pressure vessel dosimetry applications are presently freely distributed by OECD-NEADB and ORNL-RSICC and have the same energy group structure $(47 \mathrm{n}+20 \gamma)$ and general features as the ORNL DLC-0185/BUGLE-96 [6] (ENDF/B-VI.3) reference library, generated with a similar data

\footnotetext{
${ }^{a}$ Corresponding author: massimo.pescarini@enea.it
}

This is an Open Access article distributed under the terms of the Creative Commons Attribution License 2.0, which permits unrestricted use, distribution, and reproduction in any medium, provided the original work is properly cited. 
processing methodology and internationally used with success for a long time. Concerning the free availability of updated BUGLE-type libraries, it is noted that at present BUGJEFF311.BOLIB is the first and only one released library of this type based on JEFF data. Moreover BUGENDF70.BOLIB is not a duplication of the ORNL DLC-0245/BUGLE-B7 [7] library based on ENDF/B-VII.0 data since the latter was processed in a different way, i.e., it was exclusively generated through the ORNL AMPX6.1 nuclear data processing system not freely released.

BUGJEFF311.BOLIB and BUGENDF70.BOLIB were preliminarily tested, through threedimensional (3D) fixed source transport calculations performed with the ORNL TORT-3.2 [8, 9] discrete ordinates $\left(\mathrm{S}_{\mathrm{N}}\right)$ code, on the PCA-Replica 12/13 $\left(\mathrm{H}_{2} \mathrm{O} / \mathrm{Fe}\right)$ [10] and VENUS-3 [11] low-flux engineering neutron shielding benchmark experiments included in the SINBAD [12] database of reactor shielding experiments and specifically conceived to test the accuracy of nuclear data and transport codes used in LWR shielding and radiation damage analyses.

This contribution is mainly dedicated to promote the use of 3D deterministic transport codes in the cited LWR applications, permitting in parallel a consistent comparison between results obtained with different nuclear data. In fact in this kind of calculations these codes are highly competitive with the stochastic codes (e.g. the Monte Carlo codes) also when complex geometries are involved, if they are supported by dedicated pre/post-processor systems based on combinatorial geometry, as the freely released ENEA-Bologna BOT3P-5.3 $[13,14]$ system, able to provide the spatial mesh automatic generation and the geometrical model and result visualization.

\section{Library General Features and Data Processing Methodology}

The ENEA-Bologna Nuclear Data Group generated the BUGJEFF311.BOLIB and BUGENDF70. BOLIB broad-group coupled neutron/photon working cross section libraries in FIDO-ANISN format, respectively based on the JEFF-3.1.1 and the ENDF/B-VII.0 evaluated nuclear data libraries. BUGJEFF311.BOLIB and BUGENDF70.BOLIB, as already reported, have features similar to the ORNL BUGLE-96 working library and the same neutron and photon energy group structure (47 n + $20 \gamma$ ) with 47 neutron groups in the neutron energy range $1.0 \mathrm{E}-05 \mathrm{eV}-1.7332 \mathrm{E}+07 \mathrm{eV}$ and 20 photon groups in the photon energy range $1.0 \mathrm{E}+04 \mathrm{eV}-1.4 \mathrm{E}+07 \mathrm{eV}$. The ENEA libraries were produced following strictly the recommendations of the US ANS standard "Neutron and Gamma-Ray Cross Sections for Nuclear Power Plants" (ANSI/ANS-6.1.2-1999, Reapproved in 2009 [15]). They were generated in a similar way as previously performed at ORNL where BUGLE-96 was obtained through problem-dependent cross section collapsing, using the ORNL SCAMPI nuclear data processing system, from the ORNL DLC-0184/VITAMIN-B6 [6] (ENDF/B-VI.3) fine-group coupled n/ $\gamma(199 n+42 \gamma)$ multi-purpose library generated with the versions $91.94 \mathrm{M}$ and 94.15 of the LANL NJOY [16] nuclear data processing system. In fact BUGJEFF311.BOLIB and BUGENDF70.BOLIB were respectively obtained, through cross section collapsing (see Fig. 1), from the ENEA-Bologna fine-group pseudoproblem-independent cross section libraries NEA-1869/VITJEFF311.BOLIB (JEFF-3.1.1) [17] and NEA-1870/VITENDF70.BOLIB (ENDF/B-VII.0) [18] produced using the NJOY-99.259 [19] nuclear data processing system. These ENEA multi-purpose libraries in AMPX format are characterized by the same coupled $\mathrm{n} / \gamma$ energy group structure as VITAMIN-B6 and are identically based on the Bondarenko self-shielding factor method [20]. In order to perform the cross section collapsing for the generation of the BUGJEFF311.BOLIB and BUGENDF70.BOLIB libraries, it was used an updated and corrected version of the ORNL SCAMPI [21] nuclear data processing system, called "ENEA-Bologna 2007 revision of SCAMPI" [22] and currently freely available at OECD-NEADB and ORNL-RSICC. This SCAMPI revision, in particular, can read the double-precision GENDF binary data files from the NJOY-99.259 system used to generate VITJEFF311.BOLIB and VITENDF70.BOLIB, permits the cross section collapsing of the rectangular fission matrices and can calculate the total (prompt + delayed) neutron fission spectrum taking into account the delayed neutron component. 


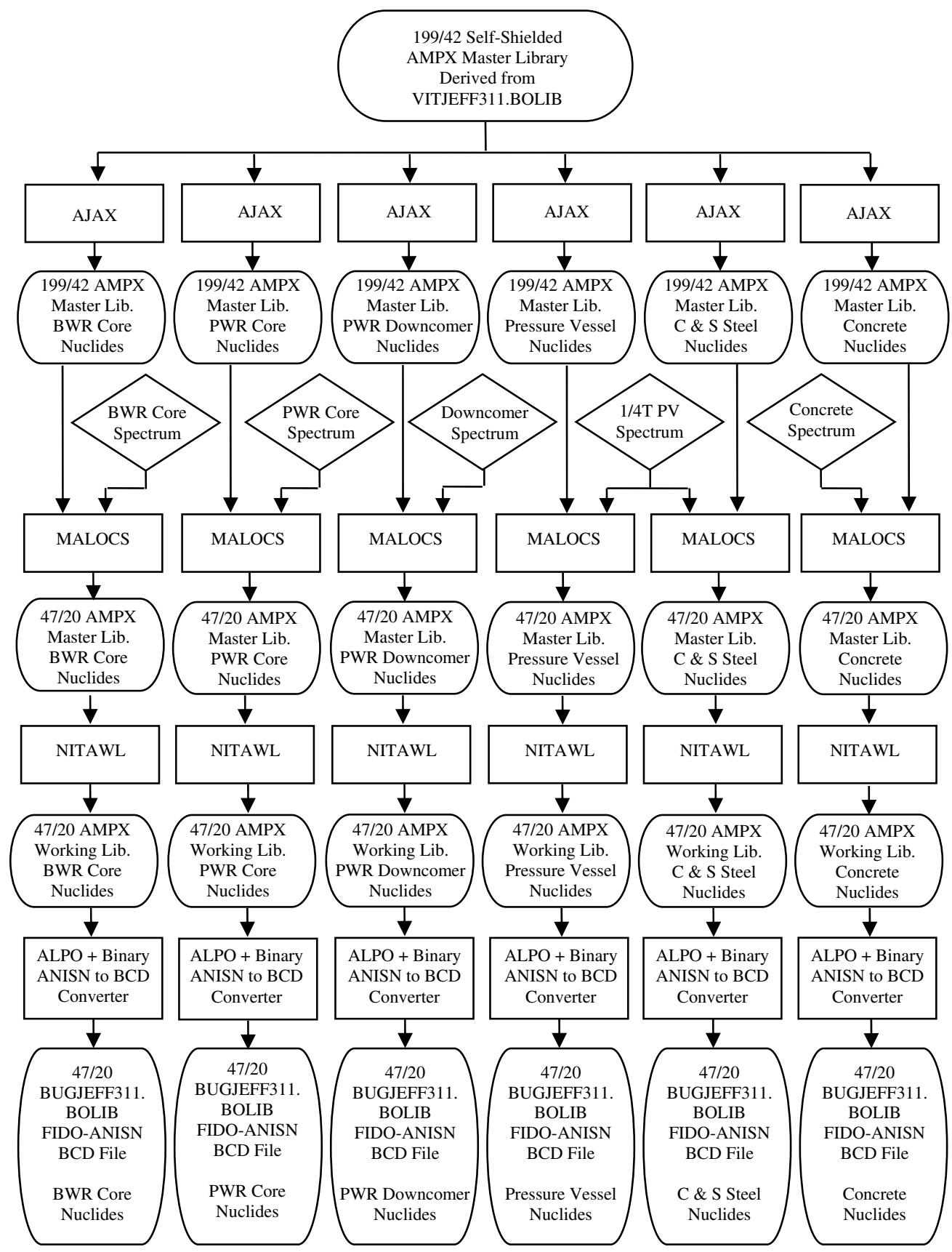

Figure 1. Procedure for generating the BUGJEFF311.BOLIB library self-shielded and collapsed cross sections using BWR- and PWR-specific flux spectra.

The ENEA BUGLE-type libraries are singularly equipped with seven blocks of cross sections. The first block includes the broad-group cross sections at the infinite dilution background cross section $\left(\sigma_{0}=1.0 \mathrm{E}+10\right.$ barns) and at the temperature of $300 \mathrm{~K}$ for all the materials (more than 180 ) contained in each corresponding ENEA VITAMIN-type mother library. These cross sections were obtained from the 
fine-group cross sections of the corresponding VITAMIN-type mother library through group collapsing with the MALOCS module of SCAMPI, using a fine-group $n / \gamma$ PWR spectrum pre-calculated in a concrete biological shield. The other six blocks contain (see [1, 2]) broad-group self-shielded cross sections which were generated from the fine-group cross sections of the corresponding VITAMIN-type mother library, self-shielded with the BONAMI module of SCAMPI at the operative temperatures and material composition background cross sections, typical of PWR and BWR material mixtures. These fine-group cross sections were then collapsed with MALOCS (see Fig. 1) using fine-group n/ $\gamma$ spectra, pre-calculated in specific representative spatial locations of five typical LWR regions: PWR core, PWR downcomer, PWR pressure vessel, reactor biological shield and BWR core. The cited specific precalculated fine-group $\mathrm{n} / \gamma$ spectra required for the cross section collapsing from each VITAMIN-type mother library were obtained from two transport calculations with the XSDRNPM one-dimensional (1D) discrete ordinates module of SCAMPI, simulating the simplified in-vessel and ex-vessel 1D radial geometries, typical of a PWR and of a BWR. The neutron spectrum calculations were performed using self-shielded neutron cross sections in the fine-group energy structure, separately derived from VITJEFF311.BOLIB and VITENDF70.BOLIB.

Concerning the six blocks of self-shielded cross sections (see Fig. 1) in each BUGLE-type library, there are in particular: 1) cross sections for PWR core materials collapsed using a PWR core spectrum, 2) cross sections for coolant materials collapsed using a PWR downcomer spectrum, 3) cross sections for PWR pressure vessel materials collapsed using a PWR pressure vessel spectrum at a depth of one-fourth (1/4 T) of the total thickness $(\mathrm{T})$ within the pressure vessel, 4) cross sections for concrete materials of a reactor biological shield collapsed using a PWR spectrum in a concrete biological shield, 5) cross sections of carbon and stainless steel materials, respectively for the LWR pressure vessels and for the LWR internals, collapsed using a PWR pressure vessel spectrum at the $1 / 4 \mathrm{~T}$ position in the pressure vessel and, finally, 6) cross sections for BWR core materials collapsed using a BWR core spectrum. The NITAWL and ALPO modules of SCAMPI were used for format conversion, respectively from the AMPX master to the AMPX working format and from the AMPX working to the ANISN format while the RADE module of SCAMPI was used to perform data consistency tests. It is underlined that the fission neutron spectra of the fissionable nuclides contained in BUGJEFF311.BOLIB and BUGENDF70.BOLIB, differently from those included in BUGLE-96 which contain only the prompt neutron spectrum component, are the total (prompt + delayed) fission neutron spectra derived from the corresponding fine-group data of VITJEFF311.BOLIB and VITENDF70.BOLIB, obtained through the ENEA-Bologna 2007 revision of SCAMPI. In addition BUGJEFF311.BOLIB and BUGENDF70.BOLIB contain response function sets for all the threshold dosimetry cross sections, directly obtained from the IAEA International Reactor Dosimetry File 2002 (IRDF-2002) [23]. The GROUPIE module of the PREPRO-2007 [24] nuclear data processing system was used to perform the data processing of the point-wise dosimetry cross section files at the temperature of $300 \mathrm{~K}$ into the BUGLE-96 47-group neutron structure. The response function dosimetry files are available in each ENEA BUGLE-type library package with two flux weightings: flat flux spectrum and 1/4 T PWR pressure vessel spectrum obtained from the previously cited PWR spectrum calculations with XSDRNPM, using alternatively VITJEFF311.BOLIB and VITENDF70.BOLIB. All the geometrical, compositional and temperature data needed for the PWR and BWR $n / \gamma$ spectra calculations and for the cross section self-shielding were consistently taken from the corresponding ORNL inputs (see [6]) of SCAMPI which generated BUGLE-96. In the ENEA-Bologna BUGLE-type libraries the maximum order of scattering available for both neutrons and photons is $\mathrm{P}_{7}$, for nuclides with $\mathrm{Z}=1$ through $\mathrm{Z}=$ 29 (copper), and $\mathrm{P}_{5}$ for the remainder of the nuclides. The versions named BUGJEFF311T.BOLIB and BUGENDF70T.BOLIB, with the thermal upscattering cross sections retained in the thermal neutron energy region below about $5.0 \mathrm{eV}$, are also included in the library packages. Concerning the ENDF/B-VII.0 based libraries, it is underlined that BUGENDF70.BOLIB was obtained through the ENEA-Bologna 2007 revision of SCAMPI from the VITENDF70.BOLIB mother library generated by 
NJOY-99.259, differently from BUGLE-B7 and its fine-group mother library VITAMIN-B7 which were both generated through AMPX-6.1.

\section{Preliminary Library Validation}

As previously reported, BUGJEFF311.BOLIB, BUGENDF70.BOLIB and BUGLE-96 were preliminarily tested using the TORT-3.2 deterministic transport code on the PCA-Replica 12/13 $\left(\mathrm{H}_{2} \mathrm{O} / \mathrm{Fe}\right)$ and VENUS-3 neutron shielding benchmark experiments.

The PCA-Replica experimental array simulates the ex-core radial geometry of a PWR with alternate regions of water and steel, including a mild steel PWR pressure vessel simulator, irradiated by a thin highly enriched $(93.0 \mathrm{w} \%$ in U-235) uranium fission plate alloyed with aluminium. Transport calculations in Cartesian (X,Y,Z) geometry and in $\mathrm{P}_{3}-\mathrm{S}_{8}$ approximation (being $\mathrm{P}_{3}$ the order of the expansion in Legendre polynomials of the scattering cross section matrix and $\mathrm{S}_{8}$ the order of the flux angular discretization) were performed using flat weighting dosimeter cross sections for the $\mathrm{Rh}$ 103(n, n')Rh-103 m, In-115(n, n')In-115 m and S-32(n,p)P-32 threshold activation dosimeters used in PCA-Replica. The calculated reaction rates for all the cited dosimeters are within the desired target accuracy of $\pm 10-15 \%$ with respect to the experimental data (see [25] for more details).

With respect to VENUS-3, a reactor with a cruciform core with typical PWR fuel assemblies and neutron spectrum, 3D transport calculations were performed in cylindrical $(\mathrm{R}, \Theta, \mathrm{Z})$ geometry, as commonly carried out in LWR pressure vessel dosimetry calculations. Fixed source calculations with one source (outer) iteration were performed in $\mathrm{P}_{3}-\mathrm{S}_{8}$ approximation. Fully symmetrical quadrature sets were introduced. The theta-weighted difference approximation was selected for the flux extrapolation model. In all the calculations the same numerical value (1.0E-04) for the point-wise flux convergence criterion was employed. The BOT3P-5.3 pre/post-processor system provided the automatic generation of the spatial meshes of the geometrical model together with the relative graphical visualizations. In particular it was described (see Fig. 2) a horizontal plane section of VENUS-3 between $0^{\circ}$ and $90^{\circ}$ (i.e. the first quadrant up to a radius of $66.0 \mathrm{~cm}$ ) containing the barrel, the neutron pad and the jacket inner wall with a $111 \times 113 \times 71(\mathrm{R}, \Theta, \mathrm{Z})$ fine spatial mesh grid. The jacket outer wall and the external regions beyond the jacket outer wall were not included since it was considered that they could only slightly affect the results. The following boundary conditions were selected: reflection at the left, inside and outside boundaries and vacuum at the right, bottom and top boundaries. The neutron source and the calculated equivalent fission fluxes, needed for the Calculated/Experimental $(\mathrm{C} / \mathrm{E})$ result comparison, were separately calculated with the consistent nuclear data library set of U-235 fission spectrum $(\chi)$ data (i.e. with BUGJEFF311.BOLIB $\chi$ total, BUGENDF70.BOLIB $\chi$ total and BUGLE-96 $\chi$ prompt data). In particular, to obtain the equivalent fission fluxes, the calculated dosimeter reaction rates (activities) were divided by the corresponding value of the flat weighting dosimeter cross section, averaged on the specific U-235 fission spectrum. The following flat weighting corresponding values (see Table 1) were used in all the calculations in order to treat simultaneously both water and steel dosimeter locations.

The VENUS-3 neutron source distribution was expressed in units of fissions per pin per second, arbitrarily normalized to a core averaged power of one fission per second per active fuel pin. Since there are 639 active pins in the simulated VENUS-3 core quadrant, including those in the partial length shield assemblies (PLSA), this normalization is equivalent to 639 fissions per second summed over the quadrant. This arbitrarily normalized source was converted to $100 \%$ of the VENUS-3 power on the basis of measured absolute fission reaction rates. In conclusion the source multiplier factor was calculated as follows: source multiplier factor $=\mathrm{N}_{\mathrm{o}} \times \bar{v}(\mathrm{U}-235) /($ quadrant volume $)=5.652 \mathrm{E}+12 \times$ $2.432 / 5.0874 \mathrm{E}+04=2.702 \mathrm{E}+08 \mathrm{n} \mathrm{cm}^{-3} \mathrm{~s}^{-1}$, where $\mathrm{N}_{\mathrm{o}}=5.652 \mathrm{E}+12$ is the measured number of fissions per second per core quadrant, corresponding to $100 \%$ of the power of the VENUS-3 core configuration, and the quadrant volume is $\cong 5.0874 \mathrm{E}+04 \mathrm{~cm}^{3}$ as deduced from the neutron source data. Finally $\bar{v}(\mathrm{U}-235)=2.432$ was the used value for the average number of neutrons produced per 

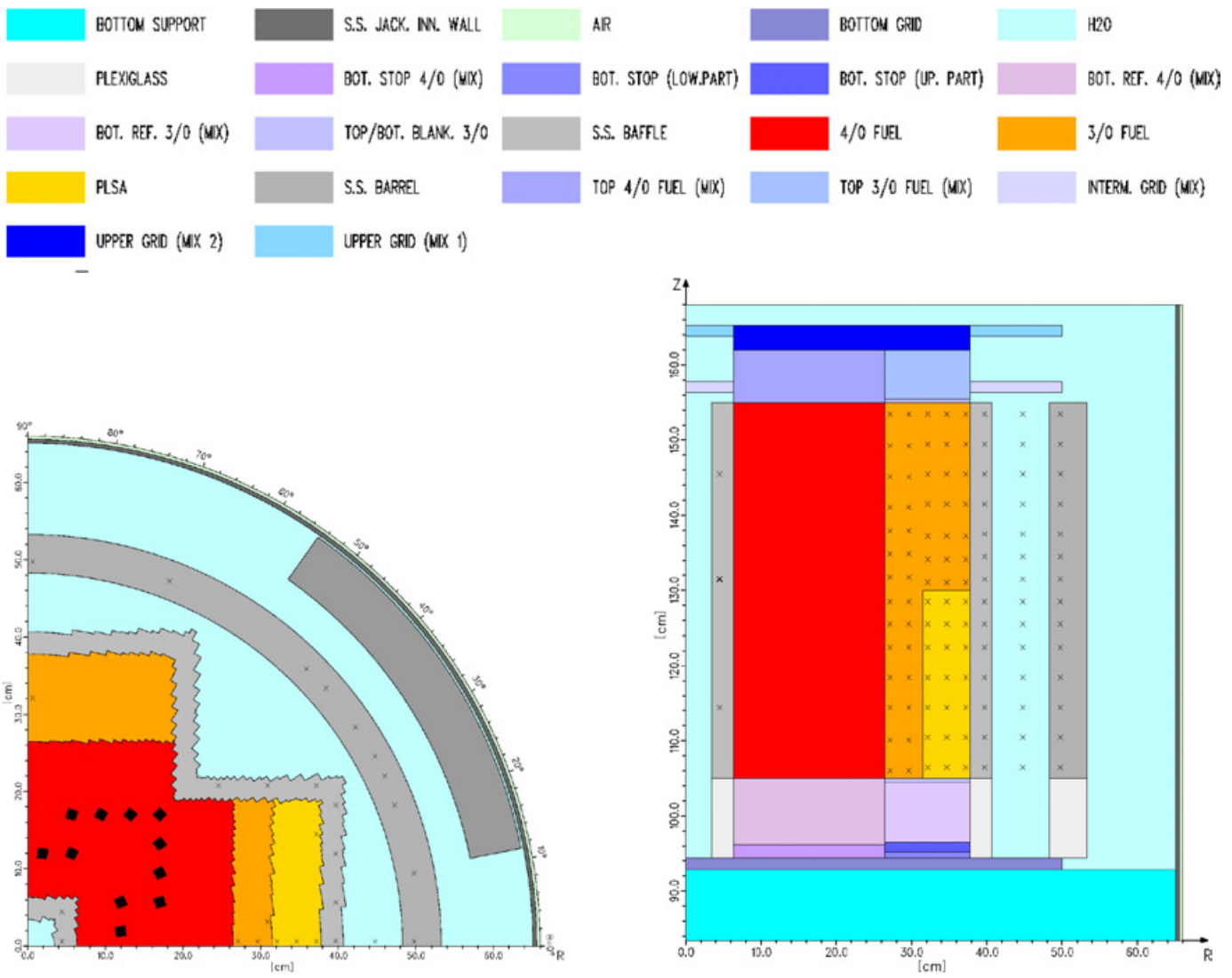

Figure 2. VENUS-3 - Geometrical and compositional model horizontal section at $\mathrm{Z}=114.50 \mathrm{~cm}$, dosimeter locations “ $\times$ ”, TORT-3.2 $(\mathrm{R}, \Theta, \mathrm{Z})$ with $111 \mathrm{R} \times 113 \Theta \times$ $71 \mathrm{Z}$ spatial meshes.

Figure 3. VENUS-3 - Geometrical and compositional model vertical section at $\Theta=0^{\circ}$, dosimeter locations “×”, TORT-3.2 $(\mathrm{R}, \Theta, \mathrm{Z})$ with $111 \mathrm{R} \times 113 \Theta \times 71 \mathrm{Z}$ spatial meshes.

U-235 thermal fission, practically identical for all the three BUGLE-type libraries. The typical dosimeter parameters in a light water material testing reactor (MTR) spectrum, similar to that of VENUS-3, are reported herewith to help to analyze the calculated results. The In-115(n, n')In-115 m, Ni-58(n,p)Co58 and Al-27(n, $\alpha$ )Na-24 dosimeters employed in VENUS-3 are respectively characterized [26] by the following effective energy thresholds of $1.30,2.26$ and $7.30 \mathrm{MeV}$ and median energies of 2.4, 3.9 and 8.6 MeV while the corrresponding $90 \%$ response energy ranges are $1.0-5.6 \mathrm{MeV}, 1.9-7.5 \mathrm{MeV}$ and 6.5-12.0 MeV. In practice the results coming from In-115(n, $\left.\mathrm{n}^{\prime}\right)$ are comparable with neutron fluxes above about $1.0 \mathrm{MeV}$ and iron DPA rates, the results from Ni-58(n,p) with neutron fluxes above about 3.0 MeV and the results from Al-27(n, $\alpha)$ with neutron fluxes above about $8.0 \mathrm{MeV}$. The corresponding flat weighting dosimeter cross sections, derived from IRDF-2002, were used in all the calculations to treat the dosimeters both in the water and steel locations. In VENUS-3 the total number of the dosimeters is 386: the In-115(n, $\left.\mathrm{n}^{\prime}\right)$ dosimeters are in 104 positions, the Ni-58(n,p) dosimeters are in 244 positions and the Al-27-(n, $\alpha)$ dosimeters are in 38 positions. In other words, each set of dosimeters is placed in a part of the 268 total different spatial locations. Axially, the dosimeters are located (see Figure 2) at 14 different axial levels between $105.0 \mathrm{~cm}$ and $155.0 \mathrm{~cm}$, respectively the lower height and the upper height of the active core region. The maximum total uncertainty of the VENUS-3 experimental 


\section{$15^{\text {th }}$ ISRD}

Table 1. VENUS-3 - IRDF-2002 dosimeter cross sections averaged on the U-235 neutron fission spectra taken from the BUGJEFF311.BOLIB, BUGENDF70.BOLIB and BUGLE-96 libraries.

\begin{tabular}{|c|c|c|c|}
\hline Library & In115 $\left(\mathrm{n}, \mathrm{n}^{\prime}\right)[\mathrm{barns}]$ & Ni-58(n,p) [barns] & Al-27(n, $\alpha)[$ barns $]$ \\
\hline BUGJEFF311.BOLIB & $1.8742 \mathrm{E}-01$ & $1.0835 \mathrm{E}-01$ & $7.8148 \mathrm{E}-04$ \\
\hline BUGENDF70.BOLIB & $1.8746 \mathrm{E}-01$ & $1.0836 \mathrm{E}-01$ & $7.8151 \mathrm{E}-04$ \\
\hline BUGLE-96 & $1.8853 \mathrm{E}-01$ & $1.0906 \mathrm{E}-01$ & $7.8671 \mathrm{E}-04$ \\
\hline
\end{tabular}

Table 2. VENUS-3 - Percentages ${ }^{\mathrm{a}}$ of the calculated (C) equivalent fission fluxes with deviations exceeding 5\% and $10 \%$ the corresponding experimental (E) ones.

\begin{tabular}{|c|c|c|c|}
\hline \multirow{2}{*}{ Library } & $\operatorname{In} 115\left(\mathrm{n}, \mathrm{n}^{\prime}\right)$ & $\mathrm{Ni}-58(n, p)$ & $\mathrm{Al}-27(\mathrm{n}, \alpha)$ \\
\hline & \multicolumn{3}{|c|}{$|\mathrm{C}-\mathrm{E}| / \mathrm{E}>5 \%$} \\
\hline BUGJEFF311.BOLIB & $4.8 \%$ & $19.7 \%$ & $28.9 \%$ \\
\hline BUGENDF70.BOLIB & $3.8 \%$ & $19.7 \%$ & $18.4 \%$ \\
\hline BUGLE-96 & $3.8 \%$ & $12.3 \%$ & $28.9 \%$ \\
\hline \multirow[b]{2}{*}{ Library } & In115(n,n') & $\mathrm{Ni}-58(n, p)$ & $\mathrm{Al}-27(\mathrm{n}, \alpha)$ \\
\hline & \multicolumn{3}{|c|}{$|\mathrm{C}-\mathrm{E}| / \mathrm{E}>10 \%$} \\
\hline BUGJEFF311.BOLIB & $0.0 \%$ & $1.2 \%$ & $0.0 \%$ \\
\hline BUGENDF70.BOLIB & $0.0 \%$ & $1.2 \%$ & $0.0 \%$ \\
\hline BUGLE-96 & $0.0 \%$ & $1.6 \%$ & $2.6 \%$ \\
\hline
\end{tabular}

$\left(^{\mathrm{a}}\right) \%$ calculated on the total number of each dosimeter type: 104 for In, 244 for Ni and 38 for $\mathrm{Al}$.

equivalent fission fluxes corresponding to each of the three dosimeters is $\pm 5 \%$. Deviations contained within $\pm 5 \%$ from the 386 dosimeter experimental data were obtained in about $83 \%$ of the calculated results with BUGJEFF311.BOLIB, $85 \%$ of the results with BUGENDF70.BOLIB and $88 \%$ of the results with BUGLE-96. The calculated equivalent fission flux results obtained with the three libraries for the 386 In-115(n, n')In-115 m, Ni-58(n,p)Co-58 and Al-27(n, $\alpha)$ Na-24 threshold activation dosimeters are within the desired target accuracy of $\pm 10-15 \%$ with respect to the experimental data (see Table 2).

The list of the main regions of the VENUS-3 calculation model are reported herewith.

- the CENTRAL HOLE (water);

- the INNER BAFFLE (stainless steel thickness: $2.858 \mathrm{~cm}$ );

- the 4/0 FUEL REGION: 4\% enriched uranium fuel rods and 11 pyrex control rods, typical of PWR poison clusters;

- the 3/0 FUEL REGION: $3.3 \%$ enriched uranium fuel rods and PLSA rods;

- the OUTER BAFFLE (stainless steel thickness. $2.858 \mathrm{~cm}$ );

- the REFLECTOR (water minimum thickness: $2.169 \mathrm{~cm}$ );

- the BARREL (stainless steel thickness: $4.99 \mathrm{~cm}$ );

- the WATER GAP (water thickness: $5.80 \mathrm{~cm}$ );

- the NEUTRON PAD (stainless steel average thickness: $6.72 \mathrm{~cm}$ );

- the VENUS environment, i.e., the jacket (air filled), the reactor vessel (stainless steel) and the reactor room (air).

\section{Conclusion}

The ENEA-Bologna freely released BUGLE-type libraries, based on OECD-NEADB JEFF-3.1.1 and US ENDF/B-VII.0 data, permitted to obtain dose results for PCA-Replica and VENUS-3 comparable with those calculated using BUGLE-96 (ENDF/B-VI.3). The desired result accuracy $( \pm 10-15 \%)$ was fully achieved but further transport analyses on neutron shielding benchmark experiments are obviously required. BUGJEFF311.BOLIB (JEFF3.1.1) is at present the only one freely released BUGLE-type 
working library based on JEFF data and permits a consistent comparison of dose results with those obtained using the BUGENDF70.BOLIB (ENDF/B-VII.0) twin library. BUGENDF70.BOLIB is not a duplication of BUGLE-B7 (ENDF/B-VII.0) since it was derived, as BUGJEFF311.BOLIB, from a mother library generated using NJOY-99.259 and from the ENEA-Bologna 2007 revision of SCAMPI whereas AMPX-6.1 (not freely released) was exclusively employed in the production of BUGLE-B7 (ENDF/B-VII.0). Anyway it seems fully confirmed the convenient applicability of modern broad-group working libraries and three-dimensional deterministic transport codes like TORT, assisted by the ENEABologna BOT3P system, to complex geometry problems. Cross section covariance data can always be added in future to the ENEA BUGLE-type libraries to perform uncertainty and sensitivity analyses, as increasingly recommended also within the activities of the industrial organizations and by OECD-NEA expert groups.

\section{References}

[1] M. Pescarini, V. Sinitsa , R. Orsi, M. Frisoni, ENEA Int. Report UTFISSM-P9H6-2 Rev.1 (2013)

[2] M. Pescarini, V. Sinitsa , R. Orsi, M. Frisoni, ENEA Int. Report UTFISSM-P9H6-8 (2013)

[3] The JEFF-3.1.1 Nuclear Data Library, JEFF Report 22, OECD-NEA Data Bank (2009)

[4] The JEFF-3.1 Nuclear Data Library, JEFF Report 21, OECD-NEA Data Bank (2006)

[5] M.B. Chadwick et al., Nuc. Data Sheets, 107, 12, pp. 2931-3060 (December 2006)

[6] J.E. White et al., ORNL-6795/R1, NUREG/CR-6214, Revision 1 (January 1995)

[7] J.M. Risner et al., ORNL/TM-2011/12, NUREG/CR-7045 (September 2011)

[8] W.A. Rhoades, D.B. Simpson, TORT Version 3, ORNL/TM-13221 (October 1997)

[9] DOORS3.1, ORNL, RSIC CCC-650 (August 1996)

[10] J. Butler et al., UKAEA, AEE Winfrith Report AEEW-R 1736 (January 1984)

[11] L. Leenders, SCK/CEN Report FCP/VEN/01 (September 1988)

[12] SINBAD, OECD-NEADB/ORNL-RSICC, SINBAD REACTOR, NEA-1517 (2009 Edition)

[13] R. Orsi, ENEA Int. Report FPN-P9H6-011 (2008)

[14] R. Orsi, Nuc. Sci. and Eng., Technical Note, 142, pp. 248-255 (2004)

[15] Am. Nat. Standard, ANSI/ANS-6.1.2-1999 (R2009) (2009)

[16] R.E. MacFarlane, D.W. Muir, The NJOY Nuclear Data Processing System, Version 91, LA12740-M (April 1994)

[17] M. Pescarini, V. Sinitsa, R. Orsi, ENEA Int. Report UTFISSM-P9H6-003 Rev.1 (2011)

[18] M. Pescarini, V. Sinitsa, R. Orsi, M. Frisoni, ENEA Int. Report UTFISSM-P9H6-005 (2012)

[19] R.E. MacFarlane, NJOY-99, "README0", ORNL, RSIC PSR-0480/02 (December 1999)

[20] I.I. Bondarenko et al., Group constants for nuclear reactors calculations (Cons. Bureau, 1964)

[21] SCAMPI, ORNL, RSIC PSR-352 (September 1995)

[22] V. Sinitsa, M. Pescarini, ENEA Int. Report FPN-P9H6-006 (2007)

[23] O. Bersillon et al., IRDF-2002, IAEA, Tech. Reports Series No. 452 (2006)

[24] D.E. Cullen, PREPRO 2007, IAEA Report IAEA-NDS-39 Rev.13 (March 2007)

[25] M. Pescarini, R.Orsi, M. Frisoni, Three Dimensional (X,Y,Z) Deterministic Analysis of the PCAReplica $\left(\mathrm{H}_{2} \mathrm{O} / \mathrm{Fe}\right)$ Neutron Shielding Benchmark Experiment Using the TORT-3.2 Code and Group Cross Section Libraries for LWR Shielding and Pressure Vessel Dosimetry, Proceedings of ISRD-15, Aix-en-Provence, F, May 18-23, 2014 (to be published)

[26] J.H. Baard, W.L. Zijp, H.J. Nolthenius, Nuclear data guide for reactor neutron metrology (Kluwer Academic Publishers, 1989) 